\title{
Multidimensional Elastic Registration of Images Using Splines
}

\author{
Jan Kybic and Michael Unser \\ Biomedical Imaging Group, IOA, DMT \\ Swiss Federal Institute of Technology Lausanne \\ CH-1015 Lausanne, Switzerland \\ Jan.Kybic@epfl.ch
}

\begin{abstract}
We present an algorithm for nonlinear multidimensional registration. The correspondence function is represented in a spline space. We also use the cubic splines to interpolate the volumetric data to be registered. We use a multiresolution strategy, which gives us robustness and additional speedup. We analyze the computational complexity of the algorithm and its performance using different multidimensional optimization methods. Finally, we present an application of the algorithm for registering ECD SPECT images for realignment of corresponding Xenon inhalation SPECT images.
\end{abstract}

\section{INTRODUCTION}

Image registration has numerous practical applications including recognition, coding, data fusion, target tracking, and others. In medical image processing, registration is used for comparing images from different modalities, different subjects, different points in time etc., see for example [1]. The registration algorithm described in this article presents several important distinguishing features: First, it is applicable to images with any number of dimensions. Second, our algorithm looks for the correspondence (or warping) function among splines, which can approximate any function with arbitrary precision by varying the step size and thus the number of parameters. Moreover, thanks to the compact support of the basis functions, the time to evaluate the criterion and its derivatives does not depend on the number of parameters describing the correspondence function. Finally, our algorithm uses a spline model also to interpolate the deformed image, which permits us to get high-order approximation and to explicitly evaluate its derivatives. The ideas used are similar to those found in [2] which uses hierarchical finite elements in the context of motion coding. It is in extension of our earlier work [3].

\section{Problem definition}

Let us consider two $N$-dimensional discrete signals $f_{r}(\mathbf{i})$ and $f_{t}(\mathbf{i})$ (where $\mathbf{i} \in \mathbb{Z}^{N}$ ), which we will call reference and test images, respectively. Suppose that these discrete images are sampled versions of continuously-defined images $f_{r}^{c}(\mathbf{x})$ and $f_{t}^{c}(\mathbf{x})$. Taking a correspondence (or warping) function $\mathbf{g}(\mathbf{x})$, we can get a warped version of the test image $f_{w}(\mathbf{x})=f_{t}^{c}(\mathbf{g}(\mathbf{x}))$. We want to find such a warping function $\mathbf{g}$, so that the warped image $f_{w}$ is as close as pos- sible to the reference image $f_{t}$, in the sense of the SSD criterion

$$
E=\sum_{\mathbf{i} \in I} e_{\mathbf{i}}^{2}=\sum_{\mathbf{i} \in I}\left(f_{w}(\mathbf{i})-f_{r}(\mathbf{i})\right)^{2}
$$

where the summation is taken over all pixels in the reference image. We generate a continuous version $f_{t}^{c}$ of the discrete image $f_{t}$ by cubic spline interpolation.

$$
f_{t}^{c}(\mathbf{x})=\sum_{\mathbf{i} \in I} b_{\mathbf{i}} \beta_{3}(\mathbf{x}-\mathbf{i})
$$

We represent the correspondence function $\mathbf{g}$ using splines as well. The model is parameterized with a moderate number of locally influent coefficients. The function $\mathbf{g}$ is a multivariate and multidimensional function $\mathbb{R}^{N} \rightarrow \mathbb{R}^{N}$.

$$
\mathbf{g}(\mathbf{x})=\mathbf{x}+\sum_{\mathbf{j} \in \mathbb{Z}^{N}} \mathbf{c}_{\mathbf{j}} \boldsymbol{\beta}_{r}(\mathbf{x} / h-\mathbf{j})
$$

where $\beta_{r}(\mathbf{x})$ is a tensor product of centered B-splines of degree $r$, that is

$$
\beta_{r}(\mathbf{x})=\prod_{k=1}^{N} \beta_{r}\left(x_{k}\right)
$$

We see that $\mathbf{g}$ is a linear combination of basis functions $\boldsymbol{\beta}_{r}$ placed on a rectangular grid. The scale parameter $h$ governs the node spacing, the total number of parameters, and the smoothness of the solution.

In some cases, the registration problem needs a regularization factor to make it well-posed, or to privilege likely solutions. Depending on the particular task, various regularizations can be justified. We investigated for example: (a) a Tikhonov stabilizer penalizing non-linear deformations

$$
E_{t}=\int \sum_{k, l, m=1}^{N}\left(\frac{\partial^{2} g_{k}}{\partial x_{l} \partial x_{m}}\right)^{2} \mathrm{~d} \mathbf{x}
$$

(b) a barrier function penalizing locally non-invertible functions

$$
E_{b}=\int \exp (-\alpha \operatorname{det}(\nabla \mathbf{g})) \mathrm{d} \mathbf{x}
$$


and (c) a very simple norm measuring the distance of $\mathbf{g}$ from identity

$$
E_{d}=\sum_{\mathbf{j}}\left\|\mathbf{c}_{\mathbf{j}}\right\|^{2}
$$

We can now formulate our problem as finding a set of coefficients c minimizing a joint criterion $E_{c}=E+\gamma E_{r}$, where $E_{r}$ is the chosen regularization factor.

\section{Optimization ALGORITHM}

We have evaluated three algorithms for solving our optimization problem: adaptive step gradient descent (GD), conjugate gradients (CG), and Marquardt-Levenberg-like regularized Newton minimization (ML). The ML method is usually the most efficient algorithm of the three in terms of the number of iterations but, as it requires evaluation of the Hessian matrix and its inversion, this does not necessarily translate into the fastest computation time.

To improve the robustness and convergence speed of the minimization, a multiresolution approach is used. The problem is first solved with coarse-resolution versions of the images. Then the solution is progressively improved at finer levels.

An example of the behavior of the three algorithms is shown in Figure 1. From this and other experiments, we found the ML method to be the most favorable.

\section{EXPLICIT DERIVATIVES}

Thanks to our spline model for both the image and the deformation function, the partial derivatives of $E$ with respect to parameters $c$ (which we need for an efficient optimization) can be found explicitly. The components of the gradient and Hessian are given by the following expressions:

$$
\begin{aligned}
\frac{\partial E}{\partial c_{\mathbf{j}, m}} & =\left.2 \sum_{\mathbf{i}} e_{\mathbf{i}} \frac{\partial f_{t}^{c}}{\partial x_{m}}\right|_{\mathbf{x}=\mathbf{g}(\mathbf{i})} \beta_{r}(\mathbf{i} / h-\mathbf{j}) \\
\frac{\partial^{2} E}{\partial c_{\mathbf{j}, m} \partial c_{\mathbf{k}, n}} & =2 \sum_{\mathbf{i}}\left(\frac{\partial f_{t}^{c}}{\partial x_{m}} \frac{\partial f_{t}^{c}}{\partial x_{n}}+\right. \\
& \left.+e_{\mathbf{i}} \frac{\partial^{2} f_{t}^{c}}{\partial x_{m} \partial x_{n}}\right)_{\mathbf{x}=\mathbf{g}(\mathbf{i})} \beta_{r}(\mathbf{i} / h-\mathbf{j}) \beta_{r}(\mathbf{i} / h-\mathbf{k})
\end{aligned}
$$

where $e_{\mathbf{i}}$ has been defined as $f_{w}(\mathbf{i})-f_{r}(\mathbf{i})$ and the partial derivatives of $f_{t}^{c}$ can be calculated from (2) simply as

$$
\left.\frac{\partial f_{t}^{c}}{\partial x_{m}}\right|_{\mathbf{x}=\mathbf{g}(\mathbf{i})}=\sum_{\mathbf{k} \in I} b_{\mathbf{k}} \beta_{3}^{\prime}\left(g_{m}(\mathbf{i})-k_{m}\right) \prod_{\substack{l=1 \\ l \neq m}}^{N} \beta_{3}\left(g_{l}(\mathbf{i})-k_{l}\right)
$$

Second-order partial derivatives are obtained in a similar fashion. As B-splines have a compact support of length 4, there is only $4^{N}$ non-zero terms in the above sum. Similarly, there is also only a limited number of non-zero terms in (8 and 9$)$.

\section{Computational complexity}

Let us give a rough estimate of the computational complexity of the registration process by counting an approximate number of arithmetic operations needed for a single evaluation of $E$, its gradient, and its Hessian. We work with images containing $n_{b}$ pixels and warping functions described by $n_{c} \times N$ parameters. The evaluation of the tensor product $\beta_{r}$ costs about $3 r N$ operations. Thus, evaluating one component of $\mathbf{g}$ at one point takes $(r+1)^{N}(3 r N+2) \approx 3 N r^{N+1}$, where $(r+1)^{N}$ is the support of $\beta_{r}$. Evaluating all $N$ components of $\mathbf{g}$ at all $n_{b}$ pixel positions costs $3 N^{2} n_{b} r^{N+1}$ operations. In the same way, we can approximate the number of operations needed to evaluate $f_{t}^{c}$ at all pixel positions, which brings the total cost of calculating $f_{w}$ to $3 N n_{b}\left(N r^{N+1}+q^{N+1}\right)$. The evaluation of $E$ adds to it $3 n_{b}$ operations.

The evaluation of the gradient and the Hessian can be optimized by elimination of common subexpressions, mainly by precalculating the values of the B-splines appearing in expressions (8) and (9), and the derivatives of $f_{t}^{c}$. The first derivatives of $f_{t}^{c}$ can be calculated in about $8 N n_{b} q^{N+1}$ operations; the B-spline values add another $3 N n_{b} r^{2}$. Since each image pixel influences only $(r+1)^{N}$ components of the gradient, thanks to the compact support of $\beta_{r}$, the final assembling of the gradient requires no more than $4 N n_{b}(r+1)^{N}$ operations.

To calculate the Hessian, second derivatives of $f_{t}^{c}$ must be calculated, which can be done in $4 N^{2} n_{b} q^{N+1}$ operations. As each image pixel influences $(r+1)^{2 N}$ components of the Hessian, the final assembling of the Hessian takes $4 N^{2} n_{b}(r+1)^{2 N}$ operations.

Note that both the gradient and Hessian evaluation costs are independent of the number of parameters. This is a very favorable feature of our method. Furthermore, the above analysis suggests that the complexity of calculating $E$, its gradient $\nabla E$, and Hessian $\nabla^{2} E$, depends linearly on the number of image pixels $n_{b}$ and nonlinearly on parameters $N, r$, and $q$. If we substitute $N=r=q=3$ into the expressions above, we find that the ratio of the three complexities are $1: 2: 40$, which corresponds very well to the timings measured practically and shown in Table I.

\section{SPECT IMAGE REGISTRATION}

We applied our algorithm to the registration of $\mathrm{ECD}^{1}$ and Xenon inhalation SPECT images [4]. These image modalities are used to visualize the blood flow in the brain. The Xenon method is non-invasive, and the resulting images contain very little anatomical information. On the other hand, the ECD method requires intravenous injection, and the resulting images show also anatomical structures in the brain. Both methods yield a 3D volume of pixels obtained by tomographic reconstruction procedure.

The position of the subjects' heads in the scanner differ as well as the head dimensions and the size of the internal structures of the brains. Therefore, in order to compare and evaluate the results of Xenon SPECT examination,

\footnotetext{
${ }^{1}$ Technetium Ethylene Cysteine Diethylester
} 

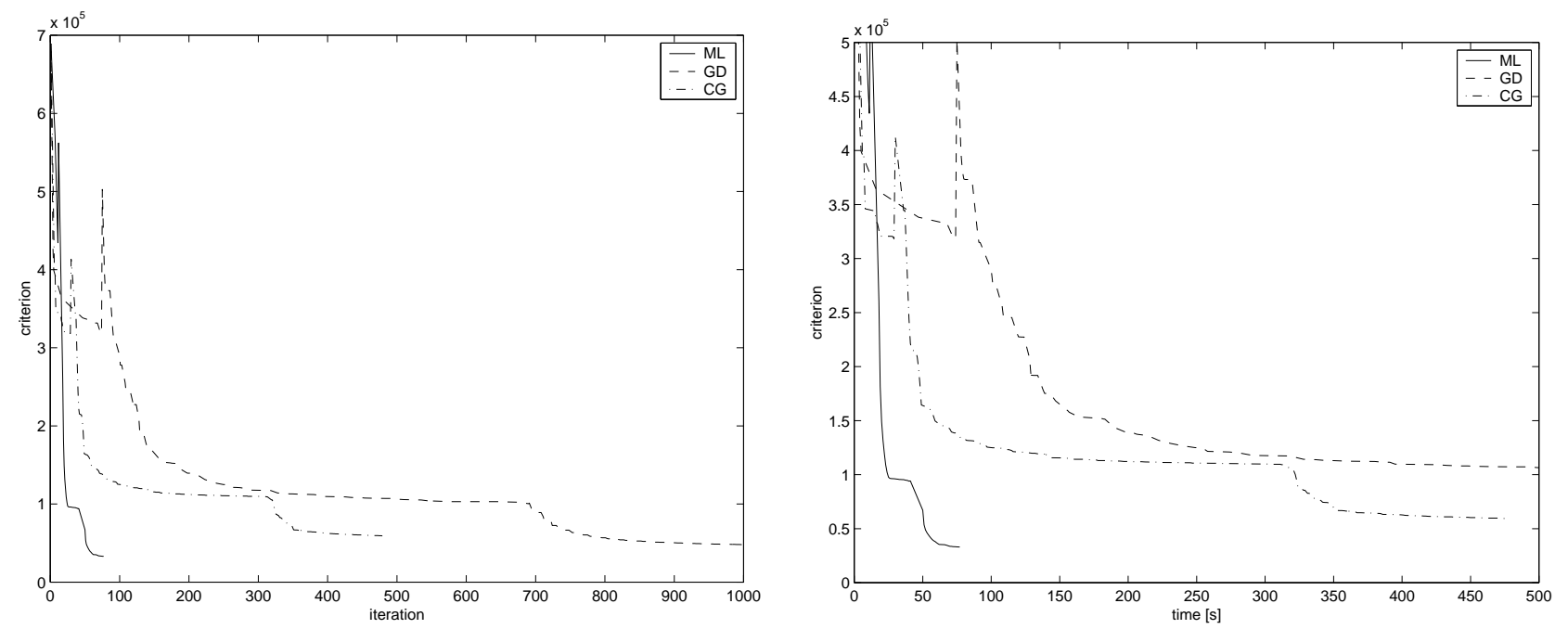

Fig. 1. Comparison of gradient descent, conjugated gradients, and Marquardt-Levenberg optimization algorithm performances. The graphs give the value of the finest-level SSD criterion of all successful (i.e., criterion-decreasing) iterations as a function of the number of criterion evaluations and execution time. The peaks are caused by transitions between resolution levels.

\begin{tabular}{|c||r|r|r|}
\hline$r$ & 2 & 3 & 3 \\
\hline$n_{c}$ & $3 \times 6 \times 6 \times 6$ & $3 \times 6 \times 6 \times 6$ & $3 \times 4 \times 4 \times 4$ \\
\hline \hline$E$ & 4.6 & 6.4 & 6.4 \\
\hline$E, \nabla E$ & 10.7 & 13.4 & 13.4 \\
\hline$E, \nabla E, \nabla^{2} E$ & 50.1 & 224.2 & 224.6 \\
\hline
\end{tabular}

TABLE I

The time in Seconds to evaluate the Criterion $E$, its gradient $\nabla E$, and Hessian $\nabla^{2} E$, for a volume of $64 \times 64 \times 17$ voxels

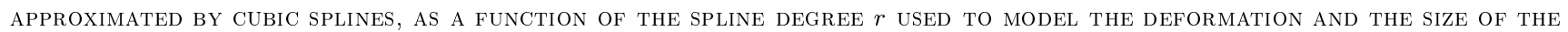
PARAMETER GRID $n_{c}$.

the volumes being compared (from different subjects) have to be registered.

We have chosen to first register the ECD SPECT images of the two subjects, and to apply the deformation found to the Xenon SPECT images. The Xenon SPECT images cannot be registered directly, because they contain too little anatomical information.

Once this method is perfected and applied to a large body of volunteers, an atlas of Xenon SPECT images will be created, permitting to use this non-invasive and fast method for diagnostical comparison of brain activities of a subject with an atlas.

As for the real application the true correspondence between the two volumes is not known and it is therefore difficult to evaluate the performance of the registration algorithm, we have chosen to test it using artificially generated random deformation, using the methodology we described in [3].

Figure 2 gives an example of the SPECT images and of the difference before and after registration for artificially generated deformation. You can see that the SPECT images are rather blurred, which augments the difficulty of the registration task. Note also that the differences in the registered images are significantly reduced. Figure 3 shows the artificially generated deformation and the resulting deformation found by our algorithm.

\section{Conclusions}

We have developed a spline registration algorithm for multiple dimensions, analyzed its computational complexity, and evaluated several solutions to the optimization problem. An important feature of our method is the multiresolution strategy. We have applied the algorithm to a practical problem in the biomedical domain.

\section{AcKnowledgments}

We are very grateful to Prof. Slosman and Dr. Chicherio from the University Hospital in Geneva for bringing this problem to our attention and for providing us with the experimental data.

\section{REFERENCES}

[1] A. W. Toga, ed., Brain Warping. San Diego: Academic Press, 1999.

[2] P. Moulin, R. Krishnamurthy, and J. Woods, "Multiscale modeling and estimation of motion fields for video coding," IEEE Transactions on Image Processing, vol. 6, Dec. 1997.

[3] J. Kybic, P. Thévenaz, A. Nirkko, and M. Unser, "Unwarping of unidirectionally distorted EPI images," IEEE Transactions on Medical Imaging, vol. 19, pp. 80-93, Feb. 2000. 

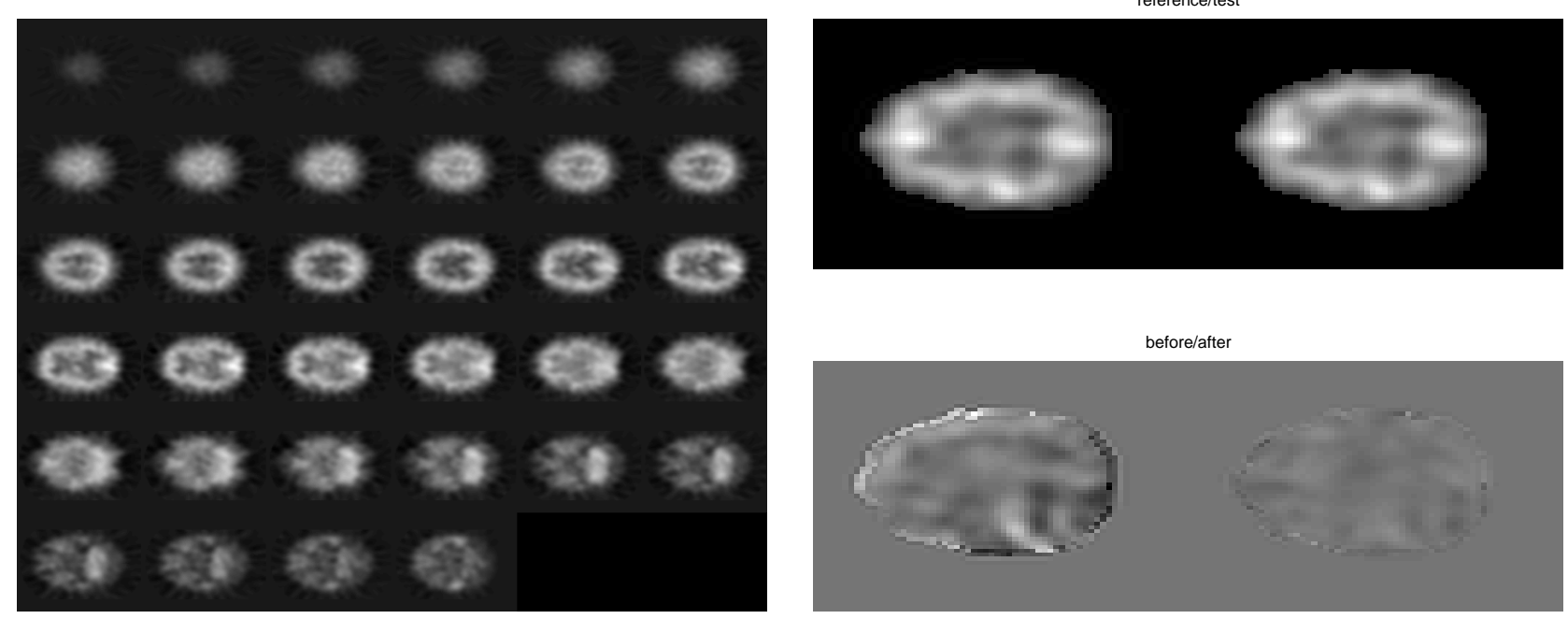

Fig. 2. The slices of an ECD SPECT image are shown on the left. One slice of the difference between and after registration of EP reconstructed Xenon SPECT images for artificially generated deformation is shown on the bottom right. The top right image shows the corresponding slices from the two EP images before registration.
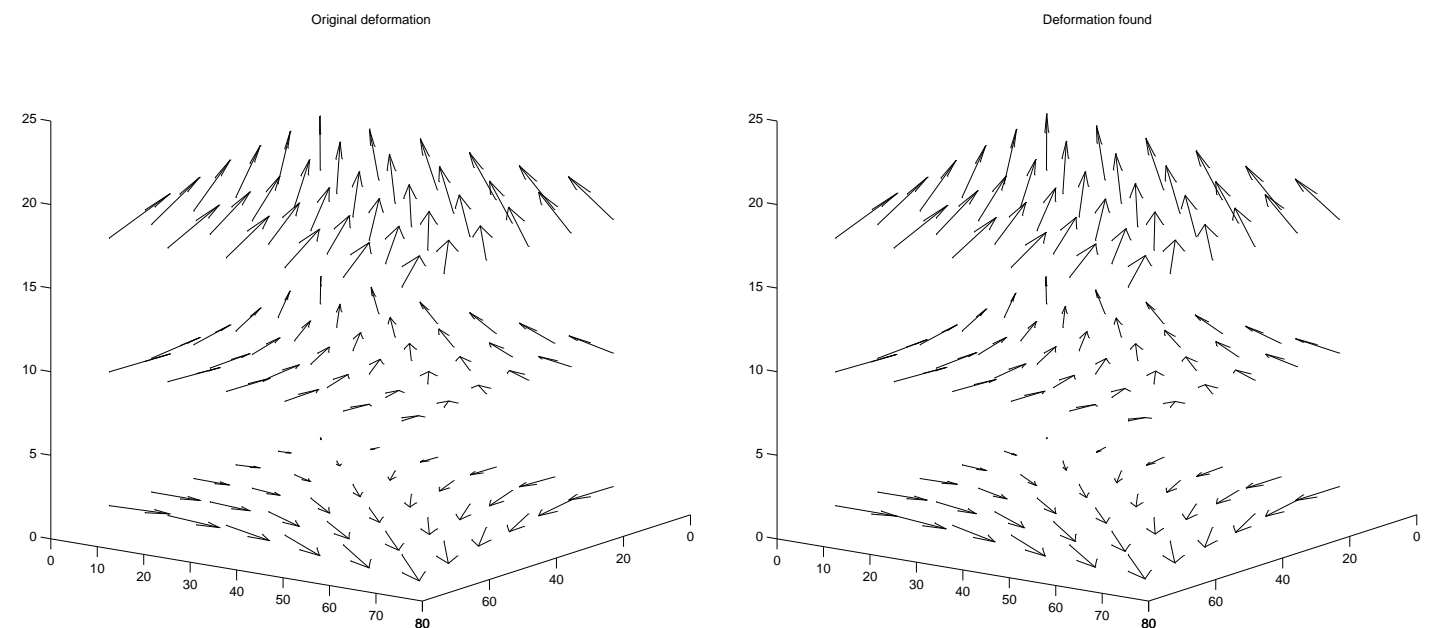

Fig. 3. The left image shows a randomly generated deformation which was applied to an ECD SPECT image in the previous figure. The right image shows the deformation recovered using our algorithm by registering the deformed SPECT image with the original one. We observe that for this model case, the deformation is very well recovered.

[4] I. Kanno and N. Lassen, "Two methods for calculating regional cerebral blood flow from emission computed tomography of inert gas concentrations," Journal of Computer Assisted Tomography, vol. 1, no. 3, pp. 71-76, 1979. 